\title{
Stability of fronts in inhomogeneous wave equations
}

\author{
Gianne Derks
}

Received: date / Accepted: date

\begin{abstract}
This paper presents an introduction to the existence and stability of stationary fronts in wave equations with finite length spatial inhomogeneities. The main focus will be on wave equations with one or two inhomogeneities. It will be shown that the fronts come in families. The front solutions provide a parameterisation of the length of the inhomogeneities in terms of the local energy of the potential in the inhomogeneity. The stability of the fronts is determined by analysing (constrained) critical points of those length functions. Amongst others, it will shown that inhomogeneities can stabilise non-monotonic fronts. Furthermore it is demonstrated that bi-stability can occur in such systems.
\end{abstract}

Keywords inhomogeneous wave equation $\cdot$ pinned front $\cdot$ stability

Mathematics Subject Classification (2000) 34D35 - 35Q53 - 37K50

\section{Introduction}

Nonlinear wave equations of the form

$$
u_{t t}=u_{x x}+V^{\prime}(u), \quad t>0, \quad x \in \mathbb{R}
$$

appear in a wide variety of applications. For example, the sine-Gordon equation with $V(u)=D(1-\cos u)$ is used to model various physical and biological systems such as superconductors, molecular systems, dislocation of crystals, and DNA processes $[3,4,8,38,39]$. Other well-studied potentials can be found in the so-called $\phi^{4}$ equation with $V(u)=\frac{1}{2} u^{2}-\frac{1}{4} u^{4}$, higher order KleinGordon potentials and the double sine-Gordon equation, see for example [3,

G. Derks

Department of Mathematics, University of Surrey, Guildford, Surrey, GU2 7XH

E-mail: g.derks@surrey.ac.uk 
7] and references in there. Note that we have chosen the convention with the plus sign in front of the potential in (1). We use this sign convention as it fits well with the spatial Hamiltonian systems that will be considered later.

A travelling wave solution is a solution of the form

$$
u(x, t)=\widehat{u}(x-c t), \text { with } \lim _{\xi \rightarrow \pm \infty} \widehat{u}^{\prime}(\xi)=0,
$$

where $\widehat{u}$ is a smooth wave shape. A stationary solution can be seen as a travelling wave with $c=0$. Substituting the travelling wave expression into (1) gives that the wave shape $\widehat{u}$ has to satisfy the ODE

$$
0=\left(1-c^{2}\right) \widehat{u}_{\xi \xi}+V^{\prime}(\widehat{u}), \xi \in \mathbb{R} ; \quad \text { and } \lim _{\xi \rightarrow \pm \infty} \widehat{u}^{\prime}(\xi)=0 .
$$

For $|c| \neq 1$, the coordinate transformation $\eta=\xi / \sqrt{\left|1-c^{2}\right|}$ gives the $c$ independent Hamiltonian ODE for the wave shape $\widehat{u}(\eta)$

$$
0=\widehat{u}_{\eta \eta}+V^{\prime}(\widehat{u}), \eta \in \mathbb{R} ; \text { and } \lim _{\eta \rightarrow \pm \infty} \widehat{u}^{\prime}(\eta)=0 .
$$

The existence and stability of travelling fronts or solitary waves in homogeneous wave equations - the potential $V$ does not depend explicitly on the spatial variable $x$ or the temporal variable $t$ - has been well studied, see for example $[7,12,29,38]$ and references therein. The existence of a travelling wave shape can be proved with dynamical systems techniques: a wave shape is a homoclinic or heteroclinic connection to fixed point(s) of the ODE for $\widehat{u}$. The smoothness of $\widehat{u}$ will depend on the smoothness of $V(u)$. If we have found one wave shape $\widehat{u}$, we get immediately a family of travelling wave solutions $u(x, t)=\widehat{u}\left(\frac{x-c t}{\sqrt{\left|1-c^{2}\right|}}\right)$ with either $|c|<1$ or $|c|>1$. The fact that travelling waves come in families is typical of Hamiltonian systems with invariance (the differential operator is Lorentz invariant) and can be related to Noether's theorem [26].

If a wave shape $\widehat{u}$ exists, we find the linear stability of the associated (travelling) waves by analysing the operator obtained by linearising about the wave shape. To find this operator, write $u(x, t)=\widehat{u}(\xi)+e^{\lambda t} v(\xi, t)$, with $\xi=x-c t$, and linearise about $v=0$. This give the eigenvalue problem

$$
\mathcal{L}_{\mathrm{f}} v=\Lambda v, \text { with } \mathcal{L}_{\mathrm{f}}=\left(1-c^{2}\right) D_{\xi \xi}+V^{\prime \prime}(\widehat{u}(\cdot)) \text { and } \Lambda=\lambda^{2} .
$$

The natural domain for $\mathcal{L}_{\mathrm{f}}$ is $H^{2}(\mathbb{R})$ as $\mathcal{L}_{\mathrm{f}}$ is derived from the wave equation (1). We call $\Lambda$ an eigenvalue of $\mathcal{L}_{\mathrm{f}}$ if there is a function $v \in H^{2}(\mathbb{R})$, which satisfies $\mathcal{L}_{\mathrm{f}} v=\Lambda v$. This operator is self-adjoint, hence all eigenvalues $\Lambda$ will be real. Furthermore, it is a Sturm-Liouville operator, thus the Sobolev Embedding Theorem gives that the eigenfunctions are continuously differentiable functions in $H^{2}(\mathbb{R})$. Sturm's Theorem [34] can be applied, leading to the fact 
that the eigenvalues are simple and bounded from above. Furthermore, if $v_{1}$ is an eigenfunction of $\mathcal{L}_{\mathrm{f}}$ with eigenvalue $\Lambda_{1}$ and $v_{2}$ is an eigenfunction of $\mathcal{L}_{\mathrm{f}}$ with eigenvalue $\Lambda_{2}$ with $\Lambda_{1}>\Lambda_{2}$, then there is at least one zero of $v_{2}$ between any pair of zeros of $v_{1}$ (including the zeros at $\pm \infty$ ). Hence, the eigenfunction $v_{1}$ has a fixed sign (no zeros) if and only if $\Lambda_{1}$ is the largest eigenvalue of $\mathcal{L}_{\mathrm{f}}$.

Assuming that $\widehat{u} \in H^{3}(\mathbb{R})$, by differentiating the ODE for $\widehat{u}$ with respect to $\xi$, we see that $\widehat{u}_{\xi}$ is an eigenfunction of $\mathcal{L}_{\mathrm{f}}$ with the eigenvalue zero. Using the Sturm-Liouville argument above: if a front/solitary wave $\widehat{u}$ exists, it is stable when it is monotonic and unstable otherwise. Nonlinear stability follows via an energy-type argument, see for example [12].

So the existence and stability of (travelling) fronts and solitary waves in homogeneous wave equations are well characterised. On the contrary, less is known about the existence and stability of fronts or solitary waves in inhomogeneous wave equations. We will focus on inhomogeneous wave equations where the potential depends on the spatial variable $x$, but not on the temporal variable $t$ :

$$
u_{t t}=u_{x x}+\frac{\partial}{\partial u} V(u ; x), \quad t>0, \quad x \in \mathbb{R} .
$$

One type of inhomogeneity that has received quite some attention are "pointwise inhomogeneities" in the form of delta functions. For example, long Josephson junctions are two superconductors sandwiching a thin insulator [2] and they are are modelled by the sine-Gordon-like potential $V(u)=\gamma u+D(1-$ $\cos u$ ), where $\gamma$ is the induced current and $D$ represents the Josephson tunnelling critical current. In an ideal uniform Josephson junction, $D$ is constant, but if there are magnetic variations, then $D$ will vary. There is a body of work, which considers the case where $D$ consists of a constant plus a sum of delta functions, see for example $[9,10,20,21,27]$. However, in various experiments, the inhomogeneities have finite length [1,30,31,32,35,36,37]. Analytically, there has been some work: in [19], the time-dependent dynamics of a travelling front, so-called (Josephson) fluxon, in the presence of a finite size defect is considered within the framework of perturbation theory ( $D$ is near 1 ); while the scattering of a fluxon on a finite length inhomogeneity is studied in $[28]$.

An inhomogeneity breaks the translation invariance and travelling fronts can interact with the inhomogeneity in various ways. If a travelling front starts very far away from the inhomogeneity and travels towards the inhomogeneity, it will travel seemingly unperturbed for a while until it approaches the inhomogeneity. Various phenomena can be observed when it approaches the inhomogeneity (see Figure 1):

- the wave can travel through the inhomogeneity while deforming for a short while, but getting its shape back afterwards; 
- the wave can bounce back and start travelling the other way around;

- the wave can get trapped in the inhomogeneity (slightly deformed) or bounce backwards and forwards in the inhomogeneity.


Fig. 1 A travelling wave interacting with an inhomogeneity: getting stuck (left), bouncing back (middle), and travelling through (right).

Looking at those interactions, it is clear that a stationary front solution plays a role in all three interactions. In this paper, we will present an introductory overview of the systematic analysis of the existence and stability of stationary waves in inhomogeneous wave equations with finite length spatial inhomogeneities. The overview is based mainly on the work in [22,23] and will highlight the main steps and key ideas in the analysis. It aims to put the reader in a good position to use the ideas and techniques in various applications. This overview presents just one approach to wave equations with spatial inhomogeneities. There are many other approaches, a few examples are indicated below as a starting point for the interested reader. These examples are by no means exhaustive, many other interesting approaches can be found in the literature.

The interaction of travelling waves with inhomogeneities is studied in the context of the trapping of fronts or solitons by inhomogeneities for the nonlinear Schrödinger / Gross-Pitaevskii equation in [33]. Here the inhomogeneities are such that they support exactly two eigenstates ("linear defect modes"). Similar ideas are used in the analysis of Nonlinear Coupled Mode equations with defects which trap gap solitons via resonant energy transfer from incoming soliton to pinned nonlinear defect mode, see [11].

Another approach can be found in the analysis of the stability of a bright soliton in an inhomogeneous nonlinear Schrödinger equations in $[17,18]$. In the first paper, a general theory for a shooting argument in the space of Lagrangian planes is developed for a large class of inhomogeneous Schrödinger equations and it is applied to a specific example in the second paper. The topological ideas were further developed for dark solitons in inhomogeneous NLS equations in $[16,24,25]$. Further references to the stability analysis of dark solitons with small inhomogeneities, can be found in these papers. 
A final example in a non-Hamiltonian context is a stability analysis of fronts in reaction-diffusion type equations with inhomogeneities using singular perturbation techniques in $[13,14,40]$.

Returning to the focus of this paper, here we consider the case in which the potential $V$ is spatially inhomogeneous in the following way. The spatial real line is split in $N+2$ disjoint open intervals $(N \geq 1)$ :

$$
\mathbb{R}=I_{\ell} \cap I_{m_{1}} \cap \ldots \cap I_{m_{N}} \cap I_{r}
$$

Here $I_{\ell}$ is the most left interval and of the form $\left(-\infty, L_{\ell}\right), I_{r}$ is the most right interval and of the form $\left(L_{r}, \infty\right)$, and $I_{m_{1}} \ldots I_{m_{N}}$ are the bounded middle intervals. The potential $V$ consists of $N+2$ smooth $\left(C^{3}\right)$ homogeneous functions $V_{\ell}(u), V_{m_{1}}(u), \ldots, V_{m_{N}}(u), V_{r}(u)$ such that $V(u, x)=V_{i}(u)$ for $x \in I_{i}$. For example, if $N=2$, i.e., there are four intervals, i.e., $\mathbb{R}=$ $\overline{\left(-\infty, L_{\ell}\right) \cup\left(L_{\ell}, L_{m}\right) \cup\left(L_{m}, L_{r}\right), \cup\left(L_{r}, \infty\right)}$, and the potential is given by

$$
V\left(u, x ; I_{\ell}, I_{m_{1}}, I_{m_{2}}, I_{r}\right)=\left\{\begin{array}{c}
V_{\ell}(u), x \in I_{\ell}=\left(-\infty, L_{\ell}\right) \\
V_{m_{1}}(u), x \in I_{m_{1}}=\left(L_{\ell}, L_{m}\right) \\
V_{m_{2}}(u), x \in I_{m_{2}}=\left(L_{m}, L_{r}\right) \\
V_{r}(u), x \in I_{r}=\left(L_{r}, \infty\right) .
\end{array}\right.
$$

Note that without loss of generality, we can use the translation invariance of the differential operators and take $L_{m}=0, L_{\ell}=-L_{1}<0$ and $L_{r}=L_{2}>0$.

We will focus on the existence and stability of stationary fronts or solitary waves of the associated damped inhomogeneous wave equation

$$
u_{t t}=u_{x x}+\frac{\partial}{\partial u} V\left(u, x ; I_{\ell}, I_{m_{1}}, \ldots, I_{m_{N}}, I_{r}\right)-\alpha u_{t},
$$

where $\alpha \geq 0$ is a constant damping coefficient. To simplify notation, we will use the term "front" to refer both to a front and a solitary wave. In the following sections, we will first show how to use phase portraits to "construct" solutions and get a characterisation of those solutions in terms of the value of the Hamiltonians in the $N$ middle intervals. This includes the introduction of length functions $L_{i}\left(h_{1}, \ldots, h_{N}\right)$, where $L_{i}$ is the length of the $i$ th middle interval and $h_{1}, \ldots h_{N}$ are the values of Hamiltonians on those intervals. This construction shows that usually there exists a plethora of solutions. With so many solutions around, the next question is: "Which ones are stable?". This is considered in the section 3. First we show that away from bifurcation points (these will be defined), the operator $\mathcal{L}_{\mathrm{f}}$ has an eigenvalue zero if and only if the determinant of the Jacobian $\frac{\partial\left(L_{1}, \ldots, L_{N}\right)}{\partial\left(h_{1}, \ldots, h_{N}\right)}$ vanishes. Next we discuss how this can be used to determine whether or not a change of stability occurs. At the end of this paper, we will consider some open problems and possible extensions to other systems. 


\section{Existence}

Stationary fronts are solutions of the ODE

$$
0=u_{x x}+\frac{\partial}{\partial u} V\left(u, x ; I_{\ell}, I_{m_{1}}, \ldots, I_{m_{n}}, I_{r}\right) .
$$

Due to the discontinuity of $V$ as function of $x$, solutions of (4) can only be expected to be $C^{1}$-smooth, even if the potentials $V_{i}(u)$ are very smooth. The continuously differentiable solutions of (4) can be constructed by using a phase plane analysis of the various Hamiltonian ODEs

$$
u_{x x}+V_{i}^{\prime}(u)=0, \quad x \in I_{i}, \quad i=\ell, m_{1}, \ldots, m_{N}, r
$$

plus boundary conditions to "match" the solutions and their derivatives at the end points of the intervals and get decay properties for $|x| \rightarrow \infty$. To get the asymptotic behaviour of a front, the outer left potential $V_{\ell}$ must have a fixed point with an unstable manifold and the right outer potential $V_{r}$ must have a fixed point with a stable manifold. As the system is Hamiltonian, the fixed points are either saddles or centres. If the potential has a local maximum, then the fixed point is a saddle and if the potential has a local minimum, then the fixed point is a centre. Thus for the existence of a stationary front it is necessary that the outer potentials $V_{\ell}$ and $V_{r}$ have a local maximum (just as for the homogeneous case), let's denote them by $u_{ \pm \infty}$. The front in the unbounded outer left interval $I_{\ell}$ is part of the unstable manifold of the fixed point $u_{-\infty}$ in $V_{\ell}$, while in the unbounded outer right interval $I_{r}$, it is part of the stable manifold of the fixed point $u_{\infty}$ in $V_{r}$.

To find all possible fronts in such systems, we don't fix the length of the bounded middle intervals, we just assume that there will be $N$ middle intervals. The only conditions on the orbits in the middle intervals $I_{m_{1}} \ldots, I_{m_{N}}$ are that they have to connect with their neighbours. So for $I_{m_{1}}$, any orbit of the $V_{m_{1}}$ dynamics that connects to the unstable manifold in the outer left interval could be used for a connection to orbits in the dynamics of $V_{m_{2}}$, and so on through all middle intervals. Finally, the orbit in the dynamics on the last middle interval must connect to the stable manifold in the outer right interval. In each middle interval, the Hamiltonian $H_{m_{i}}(u)=u_{x}^{2}+V_{m_{i}}(u)$ is constant. So every orbit is characterised by the value of this Hamiltonian; this value will be denoted by $h_{i}$. And the distance between the two connection points on an orbit in the interval $I_{m_{i}}$ can be parametrised by the values of the value of the Hamiltonians $h_{i-1}, h_{i}$, and $h_{i+1}$. Thus the length functions can be denoted as functions $L_{i}\left(h_{1}, \ldots, h_{n}\right)$.

To illustrate these ideas, we will give details for the case $N=2$, which contains all features of the general case. This section is based on [22], in which full details can be found. Multiplying (5) through by $u_{x}$, writing $p=u_{x}$, and 
integrating with respect to $x$ gives the following Hamiltonian description for the stationary fronts (recall that $\mathbb{R}=\overline{I_{\ell} \cup I_{m_{1}} \cup I_{m_{2}} \cup I_{r}}$ ):

$$
\begin{array}{ll}
h_{1}=\frac{1}{2} p^{2}+V_{m_{1}}(u), x \in I_{m_{1}} ; & V_{-}:=V_{\ell}\left(u_{-\infty}\right)=\frac{1}{2} p^{2}+V_{\ell}(u), x \in I_{\ell} ; \\
h_{2}=\frac{1}{2} p^{2}+V_{m_{2}}(u), x \in I_{m_{2}} ; & V_{+}:=V_{r}\left(u_{+\infty}\right)=\frac{1}{2} p^{2}+V_{r}(u), x \in I_{r} .
\end{array}
$$

Here $h_{1}$ and $h_{2}$ are used for the (constant) values of the Hamiltonian in the intervals $I_{m_{1}}$ respectively $I_{m_{2}}$. Furthermore, the orbits have to match. We define $\left(u_{1}, p_{1}\right)$ to be the matching point between $I_{\ell}$ and $I_{m_{1}} ;\left(u_{2}, p_{2}\right)$ the matching point between $I_{m_{1}}$ and $I_{m_{2}}$; and $\left(u_{3}, p_{3}\right)$ the matching point between $I_{m_{2}}$ and $I_{r}$. The Hamiltonian formulation (6) gives that these points can be expressed as functions of the Hamiltonian parameters $h_{i}$ :

$$
\begin{aligned}
& \frac{1}{2} p_{1}^{2}=h_{1}-V_{m_{1}}\left(u_{1}\right)=V_{-}-V_{\ell}\left(u_{1}\right) \\
& \frac{1}{2} p_{2}^{2}=h_{1}-V_{m_{1}}\left(u_{2}\right)=h_{2}-V_{m_{2}}\left(u_{2}\right) \\
& \frac{1}{2} p_{3}^{2}=h_{2}-V_{m_{2}}\left(u_{3}\right)=V_{+}-V_{r}\left(u_{3}\right) .
\end{aligned}
$$

So $\left(u_{1}, p_{1}\right)$ will be functions of $h_{1}$ only, $\left(u_{3}, p_{3}\right)$ will be functions of $h_{2}$ only, and $\left(u_{2}, p_{2}\right)$ will be functions of both $h_{1}$ and $h_{2}$. In the first line of $(7)$, the second equality defines a function $h_{1}\left(u_{1}\right)$. However, the inverse function $u_{1}\left(h_{1}\right)$ is relevant for our analysis. If, at some point $\widetilde{u}, V_{\ell}^{\prime}(\widetilde{u})=V_{m_{1}}^{\prime}(\widetilde{u})$ and the non-degeneracy condition $V_{\ell}^{\prime \prime}(\widetilde{u}) \neq V_{m_{1}}^{\prime \prime}(\widetilde{u})$ holds, then $h_{1}\left(u_{1}\right)$ has a turning point at $u_{1}=\widetilde{u}$. This implies that there will be a bound on the $h_{1}$-values for which a solution exists. In terms of the orbits in the phase portrait, if $V_{\ell}^{\prime}\left(u_{1}\right)=V_{m_{1}}^{\prime}\left(u_{1}\right)$, then the unstable manifold touches the orbit in the first middle interval at the point $\left(u_{1}, p_{1}\right)$. Thus is natural to expect that this gives a bound on the $h_{1}$ values.

These arguments show that the curve $u_{1}\left(h_{1}\right)$ is likely to have a singularity or bifurcation at any point with $V_{\ell}^{\prime}(\hat{u})=V_{m_{1}}^{\prime}(\hat{u})$. Similarly, bifurcations/singularities can be expected for the curve $p_{1}\left(h_{1}\right)$ at $p_{1}=0$ too, as $p_{1}$ is defined in terms of $p_{1}^{2}$. The other matching points will have similar bifurcation/singularity points. So for each matching point, we define the bifurcation functions $\mathscr{B}_{i}, i=0,1,2$ :

$$
\begin{aligned}
\mathscr{B}_{0}\left(h_{1}\right) & =p_{1}\left(h_{1}\right)\left[V_{m_{1}}^{\prime}\left(u_{1}\left(h_{1}\right)\right)-V_{\ell}^{\prime}\left(u_{1}\left(h_{1}\right)\right)\right], \\
\mathscr{B}_{1}\left(h_{1}, h_{2}\right) & =p_{2}\left(h_{1}, h_{2}\right)\left[V_{m_{2}}^{\prime}\left(u_{2}\left(h_{1}, h_{2}\right)\right)-V_{m_{1}}^{\prime}\left(u_{2}\left(h_{1}, h_{2}\right)\right)\right], \\
\mathscr{B}_{2}\left(h_{2}\right) & =p_{3}\left(h_{2}\right)\left[V_{r}^{\prime}\left(u_{3}\left(h_{2}\right)\right)-V_{m_{2}}^{\prime}\left(u_{3}\left(h_{2}\right)\right)\right] .
\end{aligned}
$$

If $\widetilde{h}_{1}$ and/or $\widetilde{h}_{2}$ are such that $\mathscr{B}_{i}\left(\widetilde{h}_{1}, \widetilde{h}_{2}\right)=0$, then there is the possibility of a singularity or bifurcation. In the lemmas below, the continuation of the matching points as function of $h_{1}$ and/or $h_{2}$ is discussed. More background 
and proofs of those lemmas can be found in [22]. To avoid too many technical details, we will assume that the following non-degeneracy condition holds on the potential functions.

Assumption 1 The potentials $V_{i}, i=l, m_{1}, \ldots, m_{N}, r$ are such that if at some point $\widetilde{u}$ it holds that $V_{i}^{\prime}(\widetilde{u})=V_{j}^{\prime}(\widetilde{u})$ then $V_{i}^{\prime \prime}(\widetilde{u}) \neq V_{j}^{\prime \prime}(\widetilde{u})$.

First we consider the connection between the unbounded left interval and the first middle interval and the continuation of this matching point.

Lemma 1 ([22, section 2]) Assume that there is a point $\left(\widetilde{u_{1}}, \widetilde{p_{1}}\right)$ such that the first set of matching equations in (7) are satisfied at $h_{1}=\widetilde{h}_{1}$.

- If $\mathscr{B}_{0}\left(\widetilde{h}_{1}\right) \neq 0$ then there exist a neighbourhood of $\widetilde{h}_{1}$ for which there is a unique smooth curve of left matching points $\left(u_{1}\left(h_{1}\right), p_{1}\left(h_{1}\right)\right)$ which satisfy $(7)$ and $\left(u_{1}\left(\widetilde{h}_{1}\right), p_{1}\left(\widetilde{h}_{1}\right)\right)=\left(\widetilde{u_{1}}, \widetilde{p_{1}}\right)$.

- If $\mathscr{B}_{0}\left(\widetilde{h}_{1}\right)=0$ and $\left(\widetilde{u_{1}}, \widetilde{p_{1}}\right) \neq\left(u_{-\infty}, 0\right)$ (i.e., $\left(\widetilde{u_{1}}, \widetilde{p_{1}}\right)$ is not the left endpoint), then $\widehat{h}_{1}$ is an edge point for the existence interval for left matching points $\left(u_{1}, p_{1}\right)$. At one side of $\widetilde{h}_{1}$ two solutions curves of left matching points emerge from $\left(\widetilde{u_{1}}, \widetilde{p_{1}}\right)$ and there are none at the other side. The two solutions curves form one smooth curve in the $\left(u, u_{x}\right)$-plane.

- If $\mathscr{B}_{0}\left(\widetilde{h}_{1}\right)=0$ and $\left(\widetilde{u_{1}}, \widetilde{p_{1}}\right)=\left(u_{-\infty}, 0\right)$ (i.e., the left matching point), then there are two smooth solutions curves of potential left matching points in the $\left(u, u_{x}\right)$-plane, both containing the point $\left(\widetilde{u_{1}}, \widetilde{p_{1}}\right)$. If $V_{m_{1}}^{\prime}\left(\widetilde{u_{1}}\right) \neq V_{\ell}^{\prime}\left(\widetilde{u_{1}}\right)$, then these curves can be smoothly parametrised by $h_{1}$. If $V_{m_{1}}^{\prime}\left(\widetilde{u_{1}}\right)=V_{\ell}^{\prime}\left(\widetilde{u_{1}}\right)$, then $\widetilde{h}_{1}$ is an edge of the existence interval for the left matching points $\left(u_{1}, p_{1}\right)$.

If $\left(\widetilde{u_{1}}, \widetilde{p_{1}}\right)=\left(u_{-\infty}, 0\right)$ is the left matching point, then the bifurcation will lead to two potential matching points. However, one will be on the unstable manifold, the other on the stable manifold. If the stable and unstable manifold coincide, then both these points are left matching points. If not, the point on the stable manifold cannot be a left matching point.

An analogue result holds for the connection between the unbounded right interval and the last middle interval and the continuation of the matching point $\left(u_{3}, p_{3}\right)$. The connection between two middle intervals is slightly different.

Lemma 2 ([22, section 2]) Assume that there is a point $\left(\widetilde{u_{2}}, \widetilde{p_{2}}\right)$ such that the middle set of equations of matching equations (7) are satisfied for some $h_{1}=\widetilde{h}_{1}$ and $h_{2}=\widetilde{h}_{2}$.

- If $\mathscr{B}_{1}\left(\widetilde{h}_{1}, \widetilde{h}_{2}\right) \neq 0$, then there exist a neighbourhood of $\left(\widetilde{h}_{1}, \widetilde{h}_{2}\right)$ in which unique smooth functions of middle matching points $\left(u_{2}\left(h_{1}, h_{2}\right), p_{2}\left(h_{1}, h_{2}\right)\right)$ can be defined which satisfy $(7)$ and $\left(u_{2}\left(\widetilde{h}_{1}, \widetilde{h}_{2}\right), p_{2}\left(\widetilde{h}_{1}, \widetilde{h}_{2}\right)\right)=\left(\widetilde{u_{2}}, \widetilde{p_{2}}\right)$.

- If $\mathscr{B}_{1}\left(\widetilde{h}_{1}, \widetilde{h}_{2}\right)=0$ and $\left(\widetilde{u_{2}}, \widetilde{p_{2}}\right)$ is not a fixed point of the $V_{m_{1}}$-dynamics nor of the $V_{m_{2}}$-dynamics (i.e., if $\widetilde{p_{2}}=0$, then $V_{m_{1}}^{\prime}\left(\widetilde{u_{2}}\right) \neq 0$ and $V_{m_{2}}^{\prime}\left(\widetilde{u_{2}}\right) \neq 0$ ), then nearby $\widetilde{h}_{1}$, then there exist unique smooth curves $\widetilde{u}_{m}^{2}\left(h_{1}\right), \widetilde{p}_{m}^{2}\left(h_{1}\right)$, and 
$\widetilde{h}_{2}\left(h_{1}\right)$ such that the matching equations $(7)$ are satisfied, $\mathscr{B}_{1}\left(h_{1}, \widetilde{h}_{2}\left(h_{1}\right)\right)=$ 0 , and $\widetilde{u}_{m}^{2}\left(\widetilde{h}_{1}\right)=\widetilde{u}, \widetilde{p}_{m}^{2}\left(\widetilde{h}_{1}\right)=\widetilde{p}$, and $\widetilde{h}_{2}\left(\widetilde{h}_{1}\right)=\widetilde{h}_{2}$. Furthermore, the curve $\widetilde{h}_{2}\left(h_{1}\right)$ is bijective, hence near $\widetilde{h}$ there also exist unique smooth curves $\widetilde{u}_{m}^{1}\left(h_{2}\right), \widetilde{p}_{m}^{1}\left(h_{2}\right)$, and $\widetilde{h}_{1}\left(h_{2}\right)$ satisfying the criteria above and $\widetilde{h}_{1}\left(\widetilde{h}_{2}\left(h_{1}\right)\right)=$ $h_{1}, \widetilde{u}_{m}^{1}\left(\widetilde{h}_{2}\left(h_{1}\right)\right)=\widetilde{u}_{m}^{2}\left(h_{1}\right)$, etc.

Finally, the curve $\widetilde{h}_{2}\left(h_{1}\right)$ (or equivalently $\widetilde{h}_{1}\left(h_{2}\right)$ ) forms an edge of the existence region for middle matching points $\left(u_{2}, p_{2}\right)$. For each fixed $h_{1}$ : if $h_{2}$ is at one side of $\widetilde{h}_{2}\left(h_{1}\right)$, then two solutions curves of left matching points emerge from $\left(\widetilde{u_{2}}, \widetilde{p_{2}}\right)$ and there are none at the other side. As before, these two solutions curves form one smooth curve in the $\left(u, u_{x}\right)$-plane.

The case that $\mathscr{B}_{1}\left(\widetilde{h}_{1}, \widetilde{h}_{2}\right)=0$ and $\left(\widetilde{u_{2}}, \widetilde{p_{2}}\right)$ is a fixed point of the $V_{m_{1}}$-dynamics or the $V_{m_{2}}$-dynamics is highly degenerate and will not be considered here. For more details, see [22]. The condition that $\left(u_{2}, p_{2}\right)$ is not a fixed point of the $V_{m_{i}}$-dynamics, $i=1,2$, can be rephrased as the condition that the derivative of the front $u_{x}(x ; g, h)$ does not have a non-simple zero in the middle intervals.

Now that the matching points are fully characterised, the length functions can be defined in terms of $h_{1}$ and $h_{2}$. We will assume that there exists a connected set in the $\left(h_{1}, h_{2}\right)$ parameter space such that the matching point functions $\left(u_{i}\left(h_{1}, h_{2}\right), p_{i}\left(h_{1}, h_{2}\right)\right)$, for $i=1,2,3$, are well-defined for all $\left(h_{1}, h_{2}\right)$ values in this set. The lemmas above give that one (or more) of the bifurcation functions $\mathscr{B}_{i}$ vanishes at the $\left(h_{1}, h_{2}\right)$-values on the boundary of this set. In the $\left(u, u_{x}\right)$ phase space, the front solutions follow the $h_{1}$ - and $h_{2}$-orbits (in the $V_{m_{1}}$ resp. $V_{m_{2}}$-dynamics) and connect at the matching points $\left(u_{i}, p_{i}\right), i=1,2,3$. The length functions $L_{1}$ and $L_{2}$ are determined by the "time of flight" along these orbits. To find expressions for $L_{1}$ and $L_{2}$, we define functions $p_{1}\left(u, h_{1}\right)$ and $p_{2}\left(u, h_{2}\right)$ to satisfy

$$
\begin{aligned}
{\left[p_{1}\left(u, h_{1}\right)\right]^{2} } & =2\left[h_{1}-V_{m_{1}}(u)\right], \text { for } u \in\left\{u\left(x ; h_{1}, h_{2}\right): x \in \overline{I_{m_{1}}}\right\} \\
{\left[p_{2}\left(h_{1}, h_{2}\right)\right]^{2} } & =2\left[h_{2}-V_{m_{2}}(u)\right], \text { for } u \in\left\{u\left(x ; h_{1}, h_{2}\right): x \in \overline{I_{m_{2}}}\right\} .
\end{aligned}
$$

This implies that $p_{1}\left(u\left(x ; h_{1}, h_{2}\right), h_{1}\right)=u_{x}\left(x ; h_{1}, h_{2}\right)$, for any $x \in I_{m_{1}}$, and $p_{2}\left(u\left(x ; h_{1}, h_{2}\right), h_{2}\right)=u_{x}\left(x ; h_{1}, h_{2}\right)$, for any $x \in I_{m_{2}}$. Finally, the sign of $p_{i}$ is determined by the position of $u(x)$ on the orbit: if $u$ is increasing then $p_{i}$ is defined to be positive and if $u$ is decreasing $p_{i}$ is defined to be negative. Thus the turning points of $u$ are the points at which the sign in the definition of $p_{i}$ changes.

The expressions for the lengths $L_{i}$ depend on how many turning points the function $u\left(x ; h_{1}, h_{2}\right)$ has on the middle intervals $I_{m_{i}}$. If there are no turning points in any middle interval, then the expressions are straightforward and 
given by

$$
\begin{aligned}
& L_{1}\left(h_{1}, h_{2}\right)=\int_{0}^{L_{1}} d x=\int_{u_{1}\left(h_{1}\right)}^{u_{2}\left(h_{1}, h_{2}\right)} \frac{d u}{p_{1}\left(u, h_{1}\right)} ; \\
& L_{2}\left(h_{1}, h_{2}\right)=\int_{u_{2}\left(h_{1}, h_{2}\right)}^{u_{3}\left(h_{2}\right)} \frac{d u}{p_{2}\left(u, h_{2}\right)} .
\end{aligned}
$$

However, if the function $u\left(x ; h_{1}, h_{2}\right)$ has turning points in one or both middle intervals, the integrals have to be split at the turning points. Denoting the turning points of $u\left(x ; h_{1}, h_{2}\right)$ in the interval $I_{m_{1}}$ by $x_{1}^{1}<\ldots<x_{n_{1}}^{1}$ and those in the interval $I_{m_{2}}$ by $x_{1}^{2}<\ldots<x_{n_{2}}^{2}$, the length functions $L_{1}\left(h_{1}, h_{2}\right)$ and $L_{2}\left(h_{1}, h_{2}\right)$ are given by

$$
\begin{aligned}
& L_{1}:=\int_{u_{1}\left(h_{1}\right)}^{u\left(x_{1}^{1} ; h_{1}\right)} \frac{d u}{p_{1}\left(u, h_{1}\right)}+\sum_{i=2}^{n_{1}} \int_{u\left(x_{i-1}^{1} ; h_{1}\right)}^{u\left(x_{i}^{1} ; h_{1}\right)} \frac{d u}{p_{1}\left(u, h_{1}\right)}+\int_{u\left(x_{n_{1}} ; h_{1}\right)}^{u_{2}\left(h_{1}, h_{2}\right)} \frac{d u}{p_{1}\left(u, h_{1}\right)} ; \\
& L_{2}:=\int_{u_{2}\left(h_{1}, h_{2}\right)}^{u\left(x_{1}^{2} ; h_{2}\right)} \frac{d u}{p_{2}\left(u, h_{2}\right)}+\sum_{i=2}^{n_{2}} \int_{u\left(x_{i-1}^{2} ; h_{2}\right)}^{u\left(x_{i}^{2} ; h_{2}\right)} \frac{d u}{p_{2}\left(u, h_{2}\right)}+\int_{u\left(x_{n_{2}} ; h_{2}\right)}^{u_{3}\left(h_{2}\right)} \frac{d u}{p_{2}\left(u, h_{2}\right)} .
\end{aligned}
$$

By differentiating these expressions for the length functions and the matching equations (7), it can be seen that the middle bifurcation function and the length functions are related:

$$
\frac{\partial L_{1}}{\partial h_{2}}\left(h_{1}, h_{2}\right)=\frac{1}{\mathscr{B}_{1}\left(h_{1}, h_{2}\right)}=\frac{\partial L_{2}}{\partial h_{1}}\left(h_{1}, h_{2}\right) .
$$

Extending the results and expressions for the length functions to the case that there are $N$ middle intervals is straightforward. And if there is only one middle interval $(N=1)$ then setting $V_{m}:=V_{m_{1}}=V_{m_{2}}, L:=L_{1}=L_{2}$, and $h:=h_{1}=h_{2}$ gives the relevant relations for the matching points, bifurcation functions, and length functions.

To illustrate the ideas above, we will show the existence of families of front solutions and find the length function curves for an example with $N=1$. More examples with $N=1$ and $N=2$ can be found in [6,22]. This example is similar to the example in [23] and can be related to long Josephson junctions with defects. Consider the following potential with one middle interval

$$
V\left(u, x ; L_{1}\right)=\left\{\begin{array}{cc}
\cos (u)+\gamma u, & |x|>L_{1} \\
\frac{1}{4}(u-c)^{2}, & |x|<L_{1} .
\end{array}\right.
$$

We will focus on $\gamma=0.1$ and $c=4$ and the fronts connecting $\left(u_{-\infty}, 0\right)$ with $\left(u_{\infty}, 0\right)$, where $u_{-\infty}=\arcsin \gamma$ and $u_{-\infty}=2 \pi+\arcsin \gamma$. Following (6), the 
relations for the left and right matching points $\left(u_{1}, p_{1}\right)$ and $\left(u_{2}, p_{2}\right)$ are

$$
\begin{aligned}
\frac{1}{2} p_{i}^{2}+\frac{1}{4}\left(u_{i}-c\right)^{2} & =h, \quad i=1,2 ; \\
\cos \left(u_{1}\right)+\gamma u_{1}+\frac{1}{2} p_{1}^{2} & =\sqrt{1-\gamma^{2}}+\gamma \arcsin (\gamma) ; \\
\cos \left(u_{2}\right)+\gamma u_{2}+\frac{1}{2} p_{2}^{2} & =\sqrt{1-\gamma^{2}}+\gamma \arcsin (\gamma)+2 \pi .
\end{aligned}
$$

From the left phase portrait in Figure 2, it can be seen that there is a minimal
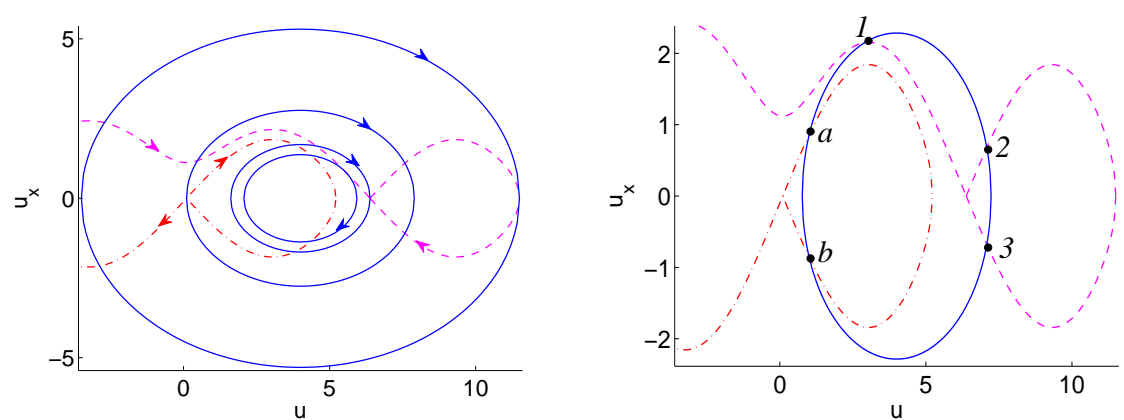

Fig. 2 Two plots of phase portraits with orbits relevant to the construction of the fronts for the example in (10) with $c=4$ and $\gamma=0.1$. In both plots, the unstable manifold of $\left(u_{-\infty}, 0\right)$ is indicated by the red dashed-dotted line; the stable manifold of $\left(u_{\infty}, 0\right)$ is the magenta dashed line; the orbits of the potential in the middle are solid blue lines.

In the left plot, the orbits for the $h$ values associated with bifurcation points are plotted. The innermost orbit is for $h=h_{\min }$, the smallest possible $h$ value for which a front exists. The next orbit is for the $h=h_{1}$, i.e. the orbit which contains the stationary point in the right interval $\left(\left(u_{\infty}, 0\right)\right)$. The third orbit is for the $h=h_{2}$, i.e., the stationary point in the left interval $\left(\left(u_{-\infty}, 0\right)\right)$ is on this orbit. And the final and outermost orbit is for $h=h_{\max }$, the largest possible value for which connections exist that use the closed part of the stable manifold.

In the right plot, an orbit for an $h$ value with the maximal number of matching points between the intervals is plotted. The possible connections between the left and middle interval are denoted by $a$ and $b$. The possible connections between the right and middle interval are denoted by 1,2 , and 3 .

value of $h$, denoted by $h_{\min }$, for which this system has at least one solution. This minimal value is associated with the solid blue orbit which touches the magenta dashed stable manifold of $\left(u_{\infty}, 0\right)$; this is the innermost blue solid orbit in Figure 2. An analysis confirms the existence of this minimal value and numerically it follows that $h_{\min } \approx 0.94$. On the orbit associated with the minimal value $h_{\min }$, there are two possible values for the left matching point. These are the points on the crossing of the red dash-dotted curve (unstable manifold of $\left.\left(u_{-\infty}, 0\right)\right)$ and the solid blue orbit associated with $h_{\min }$. They are analogues of the points denoted by $a$ and $b$ in the right plot of Figure 2 . 
The orbit associated with $h_{\text {min }}$ touches the stable manifold of $\left(u_{\infty}, 0\right)$ (dashed magenta curve), so there is exactly one right matching point. This point is the analogue of the points 1 and 3 in the right plot, at $h_{\min }$ these two points coincide. So we can conclude that there are two values for the length function $L$ at $h=h_{\min }$, see Figure 3 .

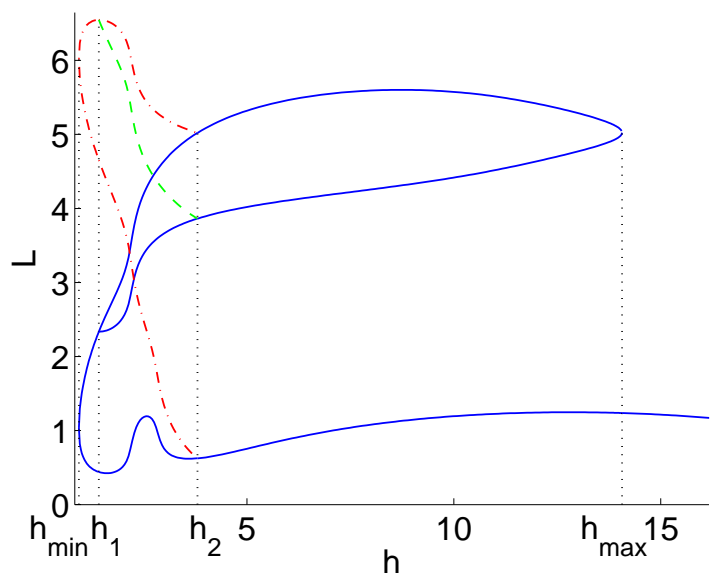

Fig. 3 The length curve for the example in (10) with $c=4$ and $\gamma=0.1$. The blue solid curve are connections involving the left matching point denoted by $a$ in Figure 2 . The red dash-dotted curve are connections of the left matching pointed denoted by $b$ with the right matching points 1 and 3. The green dashed curve are connection between $b$ and 2 .

For $h$ just above $h_{\min }$, there are two candidates for the right matching point, comparable to the the points 1 and 3 in the right plot and there are still two left matching points. Hence there are four values for the length function $L$ at those $h$ values as is also evident from Figure 3 .

If $h$ increases further, its associated orbit will cross the right asymptotic point $\left(u_{\infty}, 0\right)$. We denote this $h$ value by $h_{1} \approx 1.4$. For $h$ values just above $h_{1}$, there is a further option for the right matching point, denoted by " 2 " in the right plot of Figure 2. In the limit for $h \downarrow h_{1}$, the front associated with the right matching point " 2 " splits in the front at $h_{1}$ and the homoclinic connection to $\left(u_{\infty}, 0\right)$. So the fronts on these branches of the length function is completely disjoint from the fronts on the branches just before $h_{1}$. In Figure 3 , these two new branches are the bifurcating solid blue curve and the dashed green curve. Note that for $h>h_{1}$, the $p$ value of the point associated with " 3 " will have become negative.

Continuing further in $h$, the next "bifurcation" point is the $h$ value for which the associated orbit crossed the left asymptotic point $\left(u_{-\infty}, 0\right)$. This $h$ value is denoted by $h_{2} \approx 3.8$. At this point, the left matching points $a$ and $b$ 
merge into one and for $h>h_{2}$, only the matching point associated to $a$, which now will have $p<0$, will survive. Again, for $h \uparrow h_{2}$, the fronts associated with the left matching point "b" split in the front at $h_{2}$ and homoclinic connection to $\left(u_{-\infty}, 0\right)$

The final bifurcation point is when the orbit of the middle dynamics touches the most right point of the closed part of the stable manifold. We denote this $h$ value by $h_{\max } \approx 14$. At $h_{\max }$, the points denoted by 2 and 3 collide and for $h>h_{\max }$ these points do not exist anymore. For $h>h_{\max }$, there is exactly one left matching point ("a") and one right matching point " 1 "), both are to the left of the left asymptotic point $\left(u_{-\infty}, 0\right)$.

\section{Stability}

In the previous section, it is established that there is a large family of solutions, parametrised by the values of the Hamiltonians in the middle intervals. So a natural next question is: Which of those solutions are stable? We focus on linear stability as nonlinear stability can be deduced from this as indicated in the Introduction.

Let $\widehat{u}\left(x ; h_{1}, \ldots, h_{N}\right)$ be one of the stationary front solutions in the family. We write a solution of the wave equation (3) as $u(x, t)=\widehat{u}(x)+e^{\lambda t} v(x, t)$ and linearise about $v=0$. This gives the eigenvalue problem

$$
\mathcal{L}_{\mathrm{f}} v=\Lambda v \text {, with } \mathcal{L}_{\mathrm{f}}\left(h_{1}, \ldots, h_{N}\right)=D_{x x}+V^{\prime \prime}(\widehat{u}(\cdot)) \text { and } \Lambda=\lambda(\lambda+\alpha) .
$$

We note that $\mathcal{L}_{\mathrm{f}}$ is a symmetric operator, thus all eigenvalues $\Lambda$ are real. Since $\alpha>0$, the stability problem has a pair of solutions $\lambda_{ \pm}$with $\lambda_{+}>0$ and $\lambda_{-}<0$, if $\Lambda>0$. In other words, the stationary front is linearly unstable if $\Lambda>0$. On the other hand, the stability problem has a pair of solutions which are both strictly negative or form a complex conjugate pair with strictly negative real part if $\Lambda<0$. Therefore, the front is linearly stable if $\Lambda<0$. Finally, if $\Lambda=0$, then $\lambda=0$ or $\lambda=-\alpha$ and a change of stability will happen if $\Lambda$ is the largest eigenvalue of $\mathcal{L}_{\mathrm{f}}$ and changes sign.

Thus the eigenvalue $\Lambda$ vanishing is a necessary condition for a change of stability, but it is not sufficient as the change of stability happens only when the largest eigenvalue goes through zero. We will come back to this later.

Focusing on the question when $\Lambda=0$ is an eigenvalue, we note that $\Lambda=0$ is an eigenvalue if and only if there is a function $\Psi \in H^{2}(\mathbb{R})$ such that

$$
\mathcal{L}_{\mathrm{f}} \Psi=\Psi_{x x}+F(x) \Psi=0 \text {, where } F(x)=V_{i}^{\prime \prime}(\widehat{u}(x)), x \in I_{i},
$$

and $i=\ell, m_{1}, \ldots, m_{N}, r$. One has to be careful with the interpretation of $V^{\prime \prime}(\widehat{u}(x))$ at the end points of the middle intervals. In general there will be discontinuities and one can only consider left and right limits for $F(x)$ when 
$x$ gets to an end point of one of the middle intervals. Although differentiating the ODE for $\widehat{u}$ gives $\mathcal{L}_{\mathrm{f}} \widehat{u}_{x}=0$, this equality can not be evaluated if $x$ is an endpoint of one of the middle intervals. Moreover, usually $\widehat{u}_{x x}$ is discontinuous at those endpoints, hence $\widehat{u}_{x}$ is not in $H^{2}(\mathbb{R})$. Thus $\widehat{u}_{x}$ is not an eigenfunction with the eigenvalue zero, in contrast with the case when $V$ is homogeneous.

Although $\widehat{u}_{x}$ is not an eigenfunction, it is still a solution of the linear ODE associated with (12) on each of the open intervals $I_{\ell}, I_{r}$ and $I_{i}, i=$ $m_{1}, \ldots, m_{N}$. The theory of linear ODEs gives that on each interval there will be two linearly independent functions that span the solutions space of the ODE on this interval [15]. Using the method of variation of parameters, another linearly independent solution of the ODE can be found:

$$
x \mapsto \widehat{u}_{x}(x) \int \frac{d \xi}{\widehat{u}_{x}^{2}(\xi)} .
$$

This function is well-defined on each of the middle intervals $I_{i}$, except at points where $\widehat{u}_{x}$ vanishes. The wave shape $\widehat{u}$ satisfies a Hamiltonian ODE on each interval. Thus $\widehat{u}_{x}$ has only simple zeros or $\widehat{u}$ is associated with a fixed point of the ODE and $\widehat{u}$ is constant on the full interval. More details can be found in [22, Appendix A]. In this overview, we focus on fronts $\widehat{u}$ that are not associated with fixed points in the middle intervals and hence $\widehat{u}_{x}$ has simple zeros only at turning points of $\widehat{u}$. If $\widehat{u}$ has turning points in some middle interval, we split this interval in sub-intervals, such that the turning points are at the end points of an interval. This means that we will end up with a partition of the real line in $N_{+} \geq N+2$ intervals. We denote the endpoints of these intervals by $x_{0}<x_{1}<\ldots<x_{N_{+}}$, where $x_{0}$ is the right end point of the left interval $I_{\ell}$ and $x_{N_{+}}$the left end point of the right interval $I_{r}$. Define the mid point between $x_{i}$ and $x_{i-1}$ as $M_{i}=\frac{1}{2}\left(x_{i}+x_{i-1}\right)$. Now any solution $\Psi$ of the ODE (12) that decays at $\pm \infty$ can be written as

$$
\Psi(x)=\left\{\begin{array}{c}
\widehat{u}_{x}(x), x<x_{0} ; \\
A_{i} \widehat{u}_{x}(x)+B_{i} \widehat{u}_{x}(x) \int_{M_{i}}^{x} \frac{d \xi}{\widehat{u}_{x}^{2}(\xi)}, x_{i-1}<x<x_{i} ; \text { for } i=1, \ldots, N_{+} ; \\
\hat{k} \widehat{u}_{x}(x), x>x_{N_{+}} .
\end{array}\right.
$$

For $\Psi$ to be an eigenfunction with the eigenvalue zero, the parameters $A_{i}, B_{i}$, $i=1, \ldots, N_{+}$and $\hat{k}$ have to be such that $\Psi \in H^{2}$, hence also in $C^{1}$. The condition of continuous differentiability at all points $x_{i}, i=0, \ldots, N_{+}$leads to $2\left(N_{+}+1\right)$ conditions on the $2 N_{+}+1$ constants $A_{i}, B_{i}$ and $\hat{k}$. Thus we will get one compatibility condition for the existence of the eigenvalue zero. In [22], it is shown that this compatibility condition can be related to derivatives of the length functions $L_{i}\left(h_{1}, \ldots, h_{N}\right)$ and the following theorem is derived. 
Theorem 1 ([22, section 6]) If the solution $\widehat{u}\left(x ; h_{1}, \ldots, h_{N}\right)$ is such that $\widehat{u}_{x} \not \equiv 0$ on any middle interval, then the associated linear operator $\mathcal{L}_{\mathrm{f}}\left(h_{1}, \ldots, h_{N}\right)$ has an eigenvalue zero if and only if

$$
\operatorname{det}\left(\frac{\partial\left(L_{1}, \ldots, L_{N}\right)}{\partial\left(h_{1}, \ldots, h_{N}\right)}\right) \prod_{i=0}^{N} \mathscr{B}_{i}=0
$$

where the bifurcation functions $\mathscr{B}_{i}$ are given by

$$
\begin{aligned}
\mathscr{B}_{i}\left(h_{i-1}, h_{i}, h_{i+1}\right) & =\left[V_{i+1}^{\prime}\left(\widehat{u}\left(\chi_{i}\right)\right)-V_{i}^{\prime}\left(\widehat{u}_{i}\left(\chi_{i}\right)\right)\right] \widehat{u}_{x}\left(\chi_{i}\right), \quad i=i, \ldots, N-1 \\
\mathscr{B}_{0}\left(h_{1}\right) & =\left[V_{1}^{\prime}\left(\widehat{u}_{m}\left(\chi_{0}\right)\right)-V_{\ell}^{\prime}\left(\widehat{u}_{m}\left(\chi_{0}\right)\right)\right] \widehat{u}_{x}\left(\chi_{0}\right), \\
\mathscr{B}_{N}\left(h_{N}\right) & =\left[V_{r}^{\prime}\left(\widehat{u}\left(\chi_{N}\right)\right)-V_{N}^{\prime}\left(\widehat{u}\left(\chi_{N}\right)\right)\right] \widehat{u}_{x}\left(\chi_{N}\right)
\end{aligned}
$$

with $\chi_{i}$ denoting the endpoints of the middle intervals, i.e, $I_{m_{i}}=\left(\chi_{i-1}, \chi_{i}\right)$, $i=1, \ldots, N$.

A length function $L_{i}$ only depends on the $h$ values of its own and its adjoint intervals. Recalling that $\mathscr{B}_{i}^{-1}=\frac{\partial L_{i+1}}{\partial h_{i}}=\frac{\partial L_{i}}{\partial h_{i+1}}$ (see $\left.(9)\right)$, this implies that the Jacobian is the following tri-band matrix:

$$
\frac{\partial\left(L_{1}, \ldots, L_{N}\right)}{\partial\left(h_{1}, \ldots, h_{N}\right)}=\left(\begin{array}{ccccc}
\frac{\partial L_{1}}{\partial h_{1}} & \mathscr{B}_{1}^{-1} & 0 & \ldots & 0 \\
\mathscr{B}_{1}^{-1} & \frac{\partial L_{2}}{\partial h_{2}} & \mathscr{B}_{2}^{-1} & \ddots & 0 \\
0 & \mathscr{B}_{2}^{-1} & \ddots & \ddots & \\
\vdots & & \ddots & & \mathscr{B}_{N-1}^{-1} \\
0 & 0 & \ldots & \mathscr{B}_{N-1}^{-1} & \frac{\partial L_{N}}{\partial h_{N}}
\end{array}\right)
$$

Furthermore, when one of the bifurcation functions vanishes, this usually indicates that the boundary of the existence region has been reached and the determinant of the Jacobian goes to infinity if one approaches the boundary. However, the product of the vanishing bifurcation function and the determinant of the Jacobian, as considered in (15), is well-defined.

To further clarify criterion (15), we focus on the case when there are one or two middle intervals $(N=1$ or $N=2)$. First the case of one middle interval, i.e, there is just one parameter which we denote by $h$. Theorem 1 states that $\mathcal{L}_{\mathrm{f}}(h)$ has an eigenvalues zero if and only if

$$
\mathscr{B}_{0}(h) \mathscr{B}_{1}(h) L^{\prime}(h)=0 .
$$

It can be shown that if exactly one of the two bifurcation functions vanishes, then the limiting value of the product of the vanishing bifurcation function and the derivative $L^{\prime}(h)$ does not vanish. Altogether, Theorem 1 can be rephrased as

Theorem 2 ([22, section 4]) If $N=1$ and the solution $\widehat{u}(x ; h)$ is such that $\widehat{u}_{x} \not \equiv 0$ on the middle interval, then the associated linear operator $\mathcal{L}(h)$ has an eigenvalue zero in $H^{2}(\mathbb{R})$ if and only if $L^{\prime}(h)=0$ or $\mathscr{B}_{0}(h)=0=\mathscr{B}_{1}(h)$. 
If $\mathscr{B}_{0}(h)=0=\mathscr{B}_{1}(h)$, then the eigenfunction is a multiple of $\widehat{u}_{x}$ (possibly with different multiplication factors in each interval). And the front $\widehat{u}(h)$ is linearly stable if and only if it is strictly monotonic.

Note that the last part of this theorem recovers the results for homogeneous wave equations. In the case of a homogeneous wave equation, $V_{\ell}=V_{m}=V_{r}$, and we get immediately that $\mathscr{B}_{0}=0=\mathscr{B}_{1}$.

Next we consider the case with two middle intervals, i.e., $N=2$. Now $\mathscr{B}_{0}$ and $\mathscr{B}_{2}$ are associated with the points connecting the left respectively right intervals to the middle intervals. And $\mathscr{B}_{1}$ is associated with the connection of the two middle intervals. This is the bifurcation function that appears in the Jacobian. By analysing the special cases when one or more of the bifurcation functions vanishes, we get the following theorem.

Theorem 3 ([22, section 5]) If $\widehat{u}\left(x ; h_{1}, h_{2}\right)$ is a front solution such that all zeroes of $u_{x}(x ; g, h)$ are simple, then the associated linear operator $\mathcal{L}_{\mathrm{f}}\left(h_{1}, h_{2}\right)$ has a zero eigenvalue if and only if

$$
\mathscr{B}_{0}\left(h_{1}\right) \mathscr{B}_{1}\left(h_{1}, h_{2}\right) \mathscr{B}_{2}\left(h_{2}\right) \operatorname{det}\left(\begin{array}{cc}
\frac{\partial L_{1}}{\partial h_{1}}\left(h_{1}, h_{2}\right) & \mathscr{B}_{1}\left(h_{1}, h_{2}\right)^{-1} \\
\mathscr{B}_{1}\left(h_{1}, h_{2}\right)^{-1} & \frac{\partial L_{2}}{\partial h}\left(h_{1}, h_{2}\right)
\end{array}\right)=0 .
$$

If one or more of the bifurcation functions vanish, this expression should be read as a limit. Thus we get

- if none of the bifurcation functions vanishes, then the condition can be written as $\operatorname{det}\left(\frac{\partial\left(L_{1}, L_{2}\right)}{\partial\left(h_{1}, h_{2}\right)}\right)=0$;

- if $\mathscr{B}_{0}\left(h_{1}\right)=0, \mathscr{B}_{2}\left(h_{2}\right) \neq 0$ and $\mathscr{B}_{1}\left(h_{1}, h_{2}\right) \neq 0$, then the condition becomes $\frac{\partial L_{2}}{\partial h_{2}}\left(h_{1}, h_{2}\right)=0$;

- if $\mathscr{B}_{2}\left(h_{2}\right)=0, \mathscr{B}_{0}\left(h_{1}\right) \neq 0$ and $\mathscr{B}_{1}\left(h_{1}, h_{2}\right) \neq 0$, then the condition becomes $\frac{\partial L_{1}}{\partial h_{1}}\left(h_{1}, h_{2}\right)=0$;

- if $\mathscr{B}_{1}\left(h_{1}, h_{2}\right)=0, \mathscr{B}_{0}\left(h_{1}\right) \neq 0$ and $\mathscr{B}_{2}\left(h_{2}\right) \neq 0$, there exists a smooth bijective curve $\tilde{h}_{2}\left(h_{1}\right)$ such that $\mathscr{B}_{1}\left(h_{1}, \tilde{h}_{2}\left(h_{1}\right)\right)=0$ in a neighbourhood of this point. The curve $\tilde{h}_{2}\left(h_{1}\right)$ is bijective and a curve $\tilde{h}_{1}\left(h_{2}\right)$ can be defined such that $\tilde{h}_{1}\left(\tilde{h}_{2}\left(h_{1}\right)\right)=h_{1}$ and $\tilde{h}_{2}\left(\tilde{h}_{1}\left(h_{2}\right)\right)=h_{2}$. Condition (16) becomes

$$
0=\mathscr{B}_{0}\left(h_{1}\right) \mathscr{B}_{2}\left(h_{2}\right)\left[\frac{d L_{1}}{d h_{2}}\left(\tilde{h_{1}}\left(h_{2}\right), h_{2}\right)+\frac{d L_{2}}{d h_{1}}\left(h_{1}, \tilde{h}_{2}\left(h_{1}\right)\right)\right]
$$

- if exactly two bifurcation functions vanish, then there is no zero eigenvalue;

- if all bifurcation functions vanish, then $\mathcal{L}_{\mathrm{f}}\left(h_{1}, h_{2}\right)$ has an zero eigenvalue with the eigenfunction a multiple of $\widehat{u}_{x}$ (possibly with different multiplication factors in each interval). 
We observe that the determinant in Theorem 3 is the determinant of the Jacobian of the vector function $\left(L_{1}, L_{2}\right)\left(h_{1}, h_{2}\right)$, which is the two dimensional equivalent of $L^{\prime}(h)$, the expression in Theorem 2. Now let's consider the case of two middle intervals, but with $L_{1}$ fixed at a value $\widehat{L}$ for which $\nabla L_{1}$ does not vanish. Thus, in the existence region of the fronts, there exists a curve $\widehat{h}_{2}\left(h_{1}\right)$ or $\widehat{h}_{1}\left(h_{2}\right)$ along which $L_{1}\left(h_{1}, h_{2}\right)=\widehat{L}$. Let's focus on the case that there is a curve $\widehat{h}_{1}\left(h_{2}\right)$ (the argument for the other case is similar). Assuming that none of the bifurcation functions disappears at the fronts on this curve, we see that Theorem 3 implies that operator associated with the linearisation about a front on this curve has an eigenvalue zero if and only if the Jacobian has determinant zero. Since $L_{1}\left(\mathbf{h}\left(h_{2}\right)\right)=\widehat{L}$ for any $h_{2}$, we have $\frac{\partial L_{1}}{\partial h_{1}}\left(\mathbf{h}\left(h_{2}\right)\right) \widehat{h}_{1}^{\prime}\left(h_{2}\right)+\frac{\partial L_{1}}{\partial h_{2}}\left(\mathbf{h}\left(h_{2}\right)\right)=0$, where we used the notation $\mathbf{h}\left(h_{2}\right)=\left(\widehat{h}_{1}\left(h_{2}\right), h_{2}\right)$. Thus the Jacobian can be written as

$$
\begin{aligned}
\frac{\partial\left(L_{1}, L_{2}\right)}{\partial\left(h_{1}, h_{2}\right)}\left(\mathbf{h}\left(h_{2}\right)\right) & =\frac{\partial L_{1}}{\partial h_{1}}\left(\mathbf{h}\left(h_{2}\right)\right)\left[\frac{\partial L_{2}}{\partial h_{2}}\left(\mathbf{h}\left(h_{2}\right)\right) \frac{\partial L_{2}}{\partial h_{1}}\left(\mathbf{h}\left(h_{2}\right)\right) \widehat{h}_{1}^{\prime}\left(h_{2}\right)\right] \\
& =\frac{\partial L_{1}}{\partial h_{1}}\left(\mathbf{h}\left(h_{2}\right)\right) \frac{d}{d h_{2}} L_{2}\left(\mathbf{h}\left(h_{2}\right)\right) .
\end{aligned}
$$

Since $\nabla L_{1}$ does not vanish, this implies that the determinant vanishes if and only of $\frac{d}{d h_{2}} L_{2}\left(\mathbf{h}\left(h_{2}\right)\right)$ vanishes. So this gives us the criterion of Theorem 2 applied to the case of one middle interval being the second middle interval with the first middle interval fixed at the length $\widehat{L}$. In other words, the theorem for one middle interval can be recovered as a special case of the theorem of two middle intervals.

The theorems so far have established sufficient and necessary criteria for the existence of a zero eigenvalue. However, this does not necessarily imply a change of stability. First of all, the stability can change only if the largest eigenvalue vanishes. Since the operator $\mathcal{L}_{\mathrm{f}}$ is a Sturm-Liouville operator, the eigenfunction associated with the largest eigenvalue will have no zeros. An expression for the eigenfunction is given in (14), so this gives a method to check that the zero eigenvalue is the largest eigenvalue.

Secondly, it has to be verified that the largest eigenvalue moves through zero if the parameters are varied. Generically this will be the case, but it is also possible that the eigenvalue stays positive or negative. First we look into this issue when there is one middle interval $(N=1)$. We assume that the bifurcation functions do not vanish, i.e., we are not at the edge of the existence region. Hence $L^{\prime}(h)=0$ is the criterion for the existence of an eigenvalue zero. Assuming that the eigenvalue $\Lambda(h)$ is smooth (which is the case if the potentials in the middle and outer intervals are sufficiently smooth in $u$ ), we have the following characterisation for the local behaviour of $\Lambda(h)$ : If $\Lambda^{(k)}\left(h_{0}\right)=0$ for $k=0, \ldots, m-1$ and $\Lambda^{(m)}\left(h_{0}\right) \neq 0$ for some $m \in \mathbb{N}$, then the eigenvalue goes 
through zero if $m$ is odd and keeps the same sign if $m$ is even. By analysing the local behaviour of $\Lambda(h)$ and $L(h)$, it can be shown that the order of a zero of $\Lambda(h)$ is equal to the order of a zero of $L^{\prime}(h)$. This leads to the following necessary and sufficient criterion for a change in stability.

Theorem 4 ([23]) The stability changes at the front $\widehat{u}(\widehat{h})$ if and only if

(i) $L^{\prime}(\widehat{h})=0$ and $\widehat{h}$ is an odd zero of $L^{\prime}(h)$;

(ii) the eigenfunction $\Psi(\widehat{h})$ has no zeros.

Returning to our example in the previous section, we can use this theorem to determine the stability of the various branches by going through the following steps. First we consider the eigenfunctions at the turning points of the length curve $L(h)$. If there is an eigenfunction with no zeros, we simulate the wave equation starting nearby a front solution just away from the turning point to determine the stability of this branch. Finally we verify that the two bifurcation functions do not vanish simultaneously. These steps lead to the stability diagram as plotted in Figure 4 . The stable branches are indicated by bold curves. In this example, the stable stationary fronts with $h$ values larger than $h_{2}$ are non-monotonic. Furthermore, for all length values associated with those larger $h$ values, there are two stable fronts. Hence bi-stability is present in wave equations with a middle interval of such length.

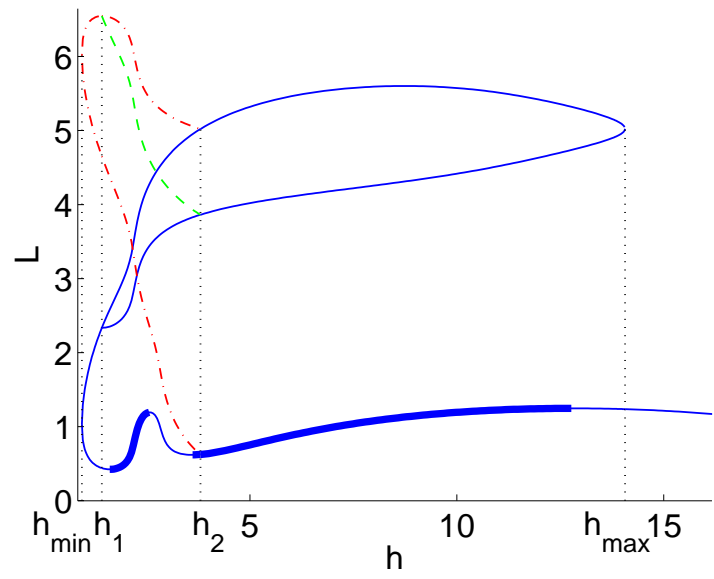

Fig. 4 The length curves for the example in (10) with $c=4$. The stationary fronts associated with the bold curves are stable, all other stationary fronts are unstable.

Theorem 4 can also be used to get a stability criterion in case there are $N$ middle intervals. Indeed, consider the case of $N$ middle intervals. Assume that there is some $\left(\widehat{h}_{1}, \ldots, \widehat{h}_{N}\right)$ in the interior of the existence region 
for which operator associated the linearisation about the front $\widehat{u}\left(\widehat{h}_{1}, \ldots, \widehat{h}_{N}\right)$ has an eigenvalue zero. Thus the Jacobian of the length function vanishes. It can be shown that the vanishing Jacobian implies that there are curves $\widetilde{h}_{2}^{1}\left(h_{1}\right), \ldots, \widetilde{h}_{N}^{1}\left(h_{1}\right)$, nearby $\left(h_{1}, \ldots, h_{N}\right)=\left(\widehat{h}_{1}, \ldots, \widehat{h}_{N}\right)$, at which the length functions satisfy $L_{j}\left(h_{1}, \widetilde{h}_{2}^{1}\left(h_{1}\right), \ldots, \widetilde{h}_{N}^{1}\left(h_{1}\right)\right)=L_{j}\left(\widehat{h}_{1}, \ldots, \widehat{h}_{N}\right), j=2, \ldots, N$. And similarly there are also curves $\widetilde{h}_{1}^{N}\left(h_{N}\right), \ldots, \widetilde{h}_{N-1}^{N}\left(h_{N}\right)$ nearby $h_{n}=\widehat{h}_{N}$ with $L_{j}\left(\widetilde{h}_{1}^{N}\left(h_{N}\right), \ldots, \widetilde{h}_{N-1}^{N}\left(h_{N}\right), h_{N}\right)=L_{j}\left(\widehat{h}_{1}, \ldots, \widehat{h}_{N}\right), j=1, \ldots, N-1$. Thus the front $\widehat{u}\left(\widehat{h}_{1}, \ldots, \widehat{h}_{N}\right)$ can be described in two different ways as a solution to a wave equation with the potential having one middle interval. One way is that the potential has $I_{m_{1}}$ as its middle interval with variable length $L_{1}$ and the other intervals are fixed at length $L_{j}\left(\widehat{h}_{1}, \ldots, \widehat{h}_{N}\right), j=2, \ldots, N$. The other way is that the potential has $I_{m_{N}}$ as its middle interval with variable length $L_{N}$ and the other intervals are fixed at length $L_{j}\left(\widehat{h}_{1}, \ldots, \widehat{h}_{N}\right), j=1, \ldots, N-1$. Applying Theorem 4 to the potentials associated with those descriptions, we get

Theorem 5 ([23]) The stability changes at the front $\widehat{u}\left(\widehat{h}_{1}, \ldots, \widehat{h}_{N}\right)$ when varying $h_{1}$ or $h_{N}$ if and only if

1. the determinant of the Jacobian $\operatorname{det}\left(\frac{\partial\left(L_{1}, \ldots, L_{N}\right)}{\partial\left(h_{1}, \ldots, h\right)}\left(\widehat{h}_{1}, \ldots, \widehat{h}_{N}\right)\right)=0$;

2. the order of the zero $\widehat{h_{1}}$ of $\frac{d}{d h_{1}}\left(L_{1}\left(h_{1}, \widetilde{h}_{2}^{1}\left(h_{1}\right), \ldots, \widetilde{h}_{N}^{1}\left(h_{1}\right)\right)\right)$ is odd or the order of the zero $\widehat{h}_{N}$ of $\frac{d}{d h_{N}}\left(L_{N}\left(\widetilde{h}_{1}^{N}\left(h_{N}\right), \ldots, \widetilde{h}_{N-1}^{N}\left(h_{N}\right), h_{N}\right)\right)$ is odd;

3. the eigenfunction $\Psi\left(\widehat{h}_{1}, \ldots, \widehat{h}_{N}\right)$ has no zeroes.

\section{Conclusions and further work}

As indicated in the Introduction, there is quite a body of work on localised inhomogeneities represented by delta functions. The delta functions are used as an approximation for very short inhomogeneities. Another approximation for this physical reality are inhomogeneities of a tiny length $L_{i}$ and potentials of the form $V_{i}(u)=u / L_{i}$. In [6], it is shown that the stationary fronts associated with localised homogeneities as described in McLAughlin \& ScotT [27] can be embedded in the family of stationary waves of finite length inhomogeneities. The stability properties follow from the theory presented in this paper and they regain the criterion derived in [27] (as well as the stability of finite length inhomogeneities).

The bi-stability observed in the example and illustrated in Figure 4 raises many interesting questions. First of all, it is likely that the bi-stability will give rise to more complicated interactions between the stationary and travelling fronts. This would be an interesting avenue for further research. Secondly, if the parameter $c$ in the example is decreased, the first maximum and the first 
minimum in the length curve will collide. Similarly for increasing $c$, the first maximum and the second minimum will collide. This is illustrated in Figure 5 for increasing $c$. The bi-stability disappears when the extrema collide, become a point of inflection (as depicted in the right plot) and the two intervals of stability merge into one.
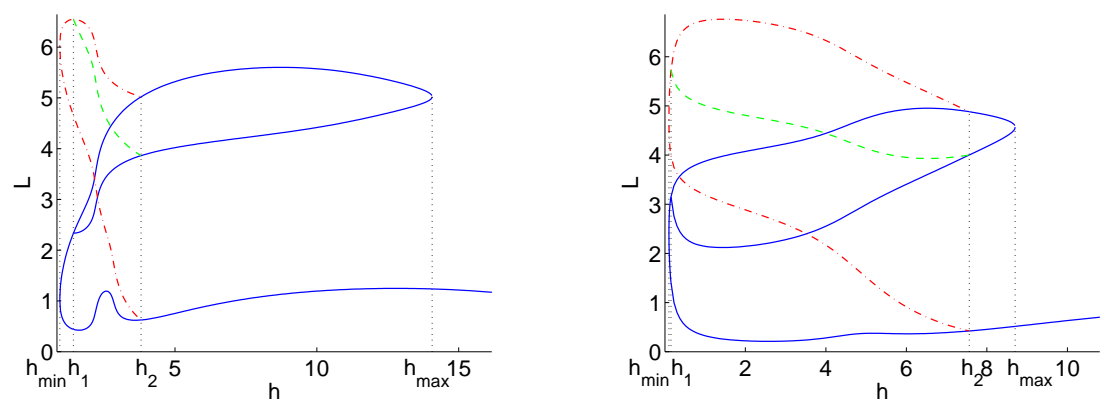

Fig. 5 The length curves for the example in (10) with $c=4$ on the left and $c=5.8$ on the right. Comparing two graphs, the first maximum and the second minimum of the graph for $c=4$ have just collided into a point of inflection at $c=5.8$ and formed an inflection point.

Another avenue for further research are coupled wave equations of the form

$$
\begin{aligned}
& u_{t t}=u_{x x}+\frac{\partial}{\partial u} V\left(u, v ; x, I_{j}\right), \\
& v_{t t}=v_{x x}+\frac{\partial}{\partial v} V\left(u, v ; x, I_{j}\right) .
\end{aligned}
$$

To prove the existence of a stationary front for one wave equation, we looked at intersections of the unstable manifold of the left endpoint with energy level sets of the middle intervals and finally matched with the stable manifold of the right endpoint. This idea is still valid, but the manifolds are higher dimensional. This makes following the intersecting manifolds more challenging. Once the existence of stationary fronts has been established, it is an open question whether the link between the stability and the vanishing determinant of the Jacobian will survive or have to be modified.

An example of the analysis of existence and stability of fronts in coupled wave equations can be found in the context of modelling the interaction between DNA and RNAP in DNA transcription [5]. Most of the stability analysis in this paper is by numerics, and it would be interesting to get analytical results. A starting point could be to use that the manifold $(u, 0)$ is invariant. Thus within this manifold we can use the existence theory of a single wave 
equation as described in the previous sections. However, the second wave equation comes into play when considering the stability of the fronts. Now stability under perturbations in the $v$-direction should be considered too.

Another direction is the existence and stability of stationary fronts just outside the invariant manifold, i.e, fronts with a tiny $v$ component. Initial results suggest that progress can be made with singular perturbation theory.

Acknowledgements I would like to thank Arjen Doelman, Stephan van Gils, Chris Knight and Hadi Susanto for sharing their enthusiasm and ideas in our investigations of wave equations with inhomogeneities.

\section{References}

1. H. Akoh, S. Sakai, A. Yagi and H. Hayakawa, Real time fluxon dynamics in Josephson transmission line, IEEE Trans. Magn. 21, pp. 737-740, 1985.

2. A. Barone, F. Esposito, C.J. Magee, A.C. Scott. Theory and applications of the sineGordon equation. Riv. Nuovo Cimento 1, 227, 1971.

3. A.R. Bishop and T. Schneider (Eds.), Solitons and Condensed Matter Physics: Proceedings of a Symposium Held June 27-29, 1978, Springer-Verlag, 1978.

4. A.S. Davydov, Solitons in Molecular Systems, Dordrecht, the Netherlands: Reidel, 1985.

5. G. Derks and G. Gaeta, A minimal model of DNA dynamics in interaction with RNAPolymerase, Physica D, 240, pp. 1805-1817, 2011.

6. G. Derks, A. Doelman, C.J.K. Knight and H. Susanto, Pinned fluxons in a Josephson junction with a finite-length inhomogeneity, European J. Appl. Math., 23(2), pp. 201244,2012

7. R.K. Dodd, J. C. Eilbeck, J. D. Gibbon and H. C. Morris, Solitons and Nonlinear Wave Equations, Academic Press, 1982.

8. J.D. Gibbon, I.N. James and I.M. Moroz, The Sine-Gordon Equation as a Model for a Rapidly Rotating Baroclinic Fluid, Phys. Script. 20, pp. 402-408, 1979.

9. R.H. Goodman and R. Haberman Interaction of sine-Gordon kinks with defects: the two bounce resonance, Physica D 195, pp 303-323, 2004.

10. R.H. Goodman and R. Haberman, Chaotic Scattering and the n-Bounce Resonance in Solitary-Wave Interactions, Phys. Rev. Lett. 98, pp 104103, 2007.

11. R.H. Goodman and Michael I. Weinstein, Stability and instability of nonlinear defect states in the coupled mode equations - analytical and numerical study, Phys. D. 237, pp. 2731-2760, 2008.

12. M. Grillakis, J. Shatah, W. Strauss. Stability theory of solitary waves in the presence of symmetry, I. Journal of Functional Analysis 74, pp.160-197, 1987.

13. P.J. van Heijster, A. Doelman, T.J. Kaper and K. Promislow, Front interactions in a three-component system, SIAM J. Appl. Dyn. Syst. 9, pp 292-332, 2010.

14. P.J. van Heijster, A. Doelman, T.J. Kaper, Y. Nishiura, K. Ueda. Pinned fronts in heterogeneous media of jump type, Nonlinearity, pp. 127-157, 2011.

15. J.K. Hale. Ordinary Differential Equations. Wiley-Interscience, 1969.

16. R. K. Jackson, R. Marangell, and H. Susanto. An instability criterion for standing waves on nonzero backgrounds. J Nonlinear Science. DOI 10.1007/s00332-01409215-8, 2014

17. C. K. R. T. Jones, Instability of standing waves for non-linear Schrödinger-type equations, Ergod. Th. \& Dynam. Sys. 8*, pp 119-138, 1988. 
18. C.K.R.T. Jones and J.V. Moloney, Instability of standing waves in nonlinear optical waveguides, Phys. Lett. A 117, pp 175-180, 1986.

19. Y.S. Kivshar, A.M. Kosevich, and O.A. Chubykalo, Finite-size effects in fluxon scattering by an inhomogeneity, Phys. Lett. A 129, pp 449-452, 1988.

20. Y.S. Kivshar and B.A. Malomed, Dynamics of solitons in nearly integrable systems, Rev. Mod. Phys. 61, pp 763-915, 1989; ibid., 63, pp 211 (Addendum), 1991.

21. Y.S. Kivshar, Zhang Fei, and L. Vázquez, Resonant soliton-impurity interactions, Phys. Rev. Lett. 67, pp 1177, 1991.

22. C.J.K. Knight, G. Derks, A. Doelman and H. Susanto Stability of Stationary Fronts in a Non-linear Wave Equation with Spatial Inhomogeneity, Journal of Differential Equations, 254, pp. 408-468, 2013.

23. C.J.K. Knight and G. Derks. A stability criterion for the non-linear wave equation with spatial inhomogeneity. Preprint, http://arxiv.org/abs/1411.5277, 2014.

24. R. Marangell, C.K.R.T. Jones, and H. Susanto, Localized standing waves in inhomogeneous Schrodinger equations, Nonlinearity 23, pp. 2059, 2010.

25. R. Marangell, H. Susanto, and C.K.R.T. Jones. Unstable Gap solitons in inhomogeneous nonlinear Schrdinger equations. Journal of Differential Equations 253, pp. 1191-1205, 2012 .

26. J.E. Marsden and T.S. Ratiu. Introduction to mechanics and symmetry, $2 \mathrm{n}$ ed.. Springer, 1999.

27. D.W. McLaughlin and A.C. Scott, Perturbation analysis of fluxon dynamics, Phys. Rev. A 18, pp. 1652-1679, 1978.

28. B. Piette and W.J. Zakrzewski, Dynamical properties of a soliton in a potential well, J. Phys. A 40, pp 329-346, 2007.

29. M.J. Rodriguez Plaza, J. Stubbe and L. Vazquez. Existence and stability of travelling waves in $(1+1)$ dimensions. J. Phys. A: Math. Gen. 23, pp. 695-705, 1990.

30. S. Sakai, H. Akoh and H. Hayakawa, Fluxon transfer devices, Japan. J. Appl. Phys. 24, p. L771, 1985.

31. S. Scharinger, C. Gürlich, R. G. Mints, M. Weides, H. Kohlstedt, E. Goldobin, D. Koelle, and R. Kleiner, Interference patterns of multifacet 20(0- $\pi$ ) Josephson junctions with ferromagnetic barrier, Phys. Rev. B 81, 174535, 2010.

32. I.L. Serpuchenko and A.V. Ustinov, Experimental observation of the fine structure on the current-voltage characteristics of long Josephson junctions with a lattice of inhomogeneities, Sov. Phys. JETP Lett. 46, p. 549 [Pis-ma Zh. Eksp. Teor. Fiz. 46, 435], 1987.

33. A. Soffer and M.I. Weinstein. Theory of nonlinear Schrödinger equations and selection of the ground state. Phys. Rev. Lett. 95, 213905, 2005.

34. E.C. Titchmarsh, Eigenfunction expansions associated with second-order differential equations, 2nd edition, Oxford University Press, 1962.

35. A.N. Vystavkin, Yu.F. Drachevskii, V.P. Koshelets, and I.L. Serpuchenko, First observation of static bound states of fluxons in long Josephson junctions with inhomogeneities, Sov. J. Low Temp. Phys. 14, pp. 357-358, 1988.

36. M. Weides, M. Kemmler, E. Goldobin, H. Kohlstedt, R. Waser, D. Koelle, and R. Kleiner, 0- $\pi$ Josephson tunnel junctions with ferromagnetic barrier, Phys. Rev. Lett. 97, p. 247001, 2006.

37. M. Weides, H. Kohlstedt, R. Waser, M. Kemmler, J. Pfeiffer, D. Koelle, R. Kleiner, and E. Goldobin, Ferromagnetic $0-\pi$ Josephson junctions, App. Phys. A 89, pp. 613-617, 2007.

38. G.B. Whitham, Linear and Nonlinear Waves, Wiley-Interscience, 1974.

39. L.V. Yakushevich, Nonlinear Physics of DNA, Wiley series in Nonlinear Science, 1998.

40. X. Yuan, T. Teramoto, and Y. Nishiura Y, Heterogeneity-induced defect bifurcation and pulse dynamics for a three-component reactiondiffusion system. Phys. Rev. E 75 $036220,2007$. 